\title{
Outbreak of scabies in a neonatal intensive care unit
}

\author{
Vini Vijayan MD² Elizabeth Marrero MSN, RN, CNOR, CIC ${ }^{2}$, Anna Gaspar MSN, RN, CCRN², Christy Wisdom MSN, RN, CIC, \\ FAPIC $^{2}$, Michele D. Honeycutt BSN, RN, BSN, $\mathrm{CIC}^{2}$ and W. Matthew Linam MD, MS ${ }^{3}$ \\ ${ }^{1}$ Pediatric Infectious Diseases Section, Department of Pediatrics, University of Arkansas for Medical Sciences, Little Rock, Arkansas, ${ }^{2}$ Infection Prevention and \\ Control Department, Arkansas Children's Hospital, Little Rock, Arkansas and ${ }^{3}$ Pediatric Infectious Diseases, Department of Pediatrics, Emory University School of \\ Medicine and Children's Healthcare of Atlanta, Atlanta, Georgia
}

Scabies outbreaks have been described in hospital settings, nursing homes, and long-term care facilities, but to our knowledge an outbreak of scabies in a neonatal intensive care unit (NICU) has not been reported previously. ${ }^{1-4}$ We report an outbreak of scabies in the NICU of a children's hospital, describe the diagnostic and treatment challenges in this subset of patients, and highlight the containment measures implemented to halt transmission.

An outbreak of scabies occurred from April through July 2016 in the 104-bed, level 4 NICU of an academic children's hospital. The index patient was a 35 -week preterm newborn admitted to the NICU due to complications of prematurity. On hospital day 54, the infant developed papules and pustules on his right arm. The rash was initially thought to be contact dermatitis, but the infant developed additional papules and pustules on the abdomen, legs and back. Due to the progression of the lesions, dermatology was consulted. On hospital day 61, skin scrapings revealed the presence of a mite, confirming the diagnosis of scabies. Failure to initially consider scabies in the differential diagnosis of the rash resulted in a 7-day delay in diagnosis, isolation, and treatment. The infant was placed on contact precautions upon diagnosis and treated with $5 \%$ permethrin lotion.

A contact investigation was performed by the infection prevention department, utilizing the following definitions. A "confirmed case" of scabies was defined as identification of mites by skin scraping and/or clinical examination. A "probable case" was defined as a person with an epidemiological link to a case and signs and symptoms consistent with scabies. Healthcare personnel (HCP) with skin-to-skin contact or close contact on multiple occasions to the index patient, and the neonates cared for by these HCP were considered exposed to scabies. Incubation period was 2-8 weeks. The attack rate was calculated by dividing the number of confirmed and probable cases by the number of exposed individuals.

The parents of the index patient were identified as the source. All exposed HCP and their household contacts underwent preemptive treatment with $5 \%$ permethrin. Of the 139 exposed neonates, 39 were $\geq 2$ months old and 100 were $<2$ months old. All 39 infants $\geq 2$ months old were treated with $5 \%$ permethrin. The 100 exposed infants $<2$ months old were not treated due to the lack of safety data for use of $5 \%$ permethrin in this age group. These infants were placed on contact precautions for the remainder of their 8 -week incubation period. The care areas for the index case and exposed patients were thoroughly cleaned,

Author for correspondence: Vini Vijayan, Email: vvijayan@uams.edu

Cite this article: Vijayan V, et al. (2019). Outbreak of scabies in a neonatal intensive care unit. Infection Control \& Hospital Epidemiology, 40: 613-614, https://doi.org/10.1017/ ice. 2019.57 and all bed linens and clothing were washed with hot water. Common family waiting areas were also cleaned. During the initial contact investigation, none of the identified exposed HCP, their household contacts, or exposed infants developed clinical signs of scabies. We later identified 3 secondary cases of scabies. This included 2 nurses and 1 occupational therapist who developed scabies 6 weeks after contact with the index case. They were not identified as exposures during the initial investigation and had not received prophylaxis. No further cases were diagnosed during the 6-month follow-up period. This resulted in an overall attack rate of $2 \%$.

This outbreak highlights the challenges encountered in the containment of a nosocomial outbreak of scabies in a NICU. In this outbreak, lack of familiarity with the clinical presentation of neonatal scabies led to a 7-day delay in diagnosis. Neonatal scabies typically presents with pustules, vesicles, or papules involving the face, scalp, palms, and soles. This presentation contrasts with that in adults, in whom intertriginous papules and burrows are classically found. The characteristic pruritus may be absent in neonates contributing to delayed diagnosis and ongoing transmission. ${ }^{5-7}$ Failure to consider scabies as the cause of a rash has been previously identified as a factor in the propagation of nosocomial outbreaks. ${ }^{1-3}$ Once considered, early and rapid diagnosis can be achieved by obtaining skin scrapings to identify the mite. ${ }^{8}$ Therefore, to facilitate prompt diagnosis and prevent spread in the NICU, the possibility of scabies should be entertained for any infant, HCP or visitor with an unexplained rash. ${ }^{9}$

During our investigation, we identified important gaps in our surveillance process. We found 3 secondary cases of scabies who had had contact to with the case patient but were not initially treated because they were missed during the initial investigation. Also, 1 HCP did not routinely work in the NICU, which hampered identification or notification of the exposure. Ensuring that all potential contacts to a case have been identified can be challenging when HCP rotate to different areas within a unit or when non-unit-based HCP provide ancillary services. Reviewing medical records for orders placed to ancillary services may help accurately identify exposed non-unit-based staff. Additionally, targeted communication to key personnel, especially those responsible for staffing, can assist in addressing this potential gap in ascertainment of contacts. This is essential because continued propagation of an outbreak can place a substantial financial burden on an institution. ${ }^{10}$ Although we did not perform a formal cost analysis in this outbreak, our institution incurred costs related to environmental cleaning and disinfection, treatment of neonates and HCP, use of isolation precautions, and hours spent on institutional control measures. 
In summary, HCP should familiarize themselves with the presentation of neonatal scabies and have a low threshold to evaluate, isolate, and treat neonates with a suspicious rash. A multifaceted approach involving clinicians, hospital administration, and environmental services is essential in preventing nosocomial scabies in the NICU setting.

Acknowledgements. None.

Financial support. No financial support was provided relevant to this article.

Conflicts of interest. All authors report no conflicts of interest relevant to this article.

\section{References}

1. Buehlmann M, Beltraminelli H, Strub C, et al. Scabies outbreak in an intensive care unit with 1,659 exposed individuals: key factors for controlling the outbreak. Infect Control Hosp Epidemiol 2009;30:354-360.
2. Ross BG, Wright-McCarthy JK, DeLaMora PA, Graham PL. Transmission of scabies in a newborn nursery. Infect Control Hosp Epidemiol 2011;32:516-517.

3. Vorou R, Remoudaki HD, Maltezou HC. Nosocomial scabies. J Hosp Infect 2007;65:9-14.

4. Hewitt KA, Nalabanda A, Cassell JA. Scabies outbreaks in residential care homes: factors associated with late recognition, burden and impact. a mixed methods study in England. Epidemiol Infect 2015;143:1542-1551.

5. Hurwitz S. Scabies in babies. Am J Dis Child 1973;126:226-228.

6. Chosidow O. Clinical practices. Scabies. N Engl J Med 2006;354:1718-1727.

7. Wilson BB. Scabies. In: Mandell GL, Bennett JE, Dolin R (editors). Mandell, Douglas and Bennett's Principles and Practice of Infectious Diseases. New York: Churchill and Livingstone; 1995:2560-2562.

8. Leung V, Miller M. Detection of scabies: a systematic review of diagnostic methods. Can J Infect Dis Med Microbiol 2011;22:143-146.

9. Nanda S, Reddy BS, Ramji S, Pandhi D. Analytical study of pustular eruptions in neonates. Pediatr Dermatol 2002;19:210-215.

10. Dik JWH, Dinkelacker AG, Verner P, et al. Cost-analysis of seven nosocomial outbreaks in an academic hospital. PLoS One 2016;11(2):e0149226. 